\title{
Letter
}

\section{The "Dynamic" Behavior of Group Psychology Represented by Culture}

\author{
Quanhua Song ${ }^{1}$, Hongji Wang ${ }^{2, *}$ \\ ${ }^{1}$ Faculty of United Front Theory, Chenzhou's Party School, Chenzhou, China \\ ${ }^{2}$ School of Economics and Finance, Guizhou University of Finance and Economics, Guiyang, China \\ Email address: \\ songquanhua0802@163.com (Quanhua Song),wanghongji1007@163.com (Hongji Wang) \\ ${ }^{*}$ Corresponding author
}

\section{To cite this article:}

Quanhua Song, Hongji Wang. The "Dynamic" Behavior of Group Psychology Represented by Culture. International Journal of Literature and Arts. Vol. 5, No. 1, 2020, pp. 6-11. doi: 10.11648/j.ijla.20200801.12

Received: December 27, 2019; Accepted: January 16, 2020; Published: January 31, 2020

\begin{abstract}
In culture and information transmission, signifier and signifier of symbols have changed into a new field with the change of ideology. For characterization of out of control and illegal ethnic composition for structural analysis of the existing culture, through the interactive relations between social groups - personal interpretation of the change of the psychological development, from the "social identification gradient" and "ideology", "the dilemma of characterization in different areas of the" three aspects to explore group psychological factors of kinetic energy release at the same time through the group of the unconscious psychological construct reshaping symbol categories, "maximum-minmum value new dynamic psychological field. Through the analysis of experience in the field of culture phenomenon, this paper proposes experience world was generated from surface world by inner-spirit selection. The main information of experience world was awaken by interactive ceremony that the public participated. Although information is an extension of the "self" image in the communication, the mirror is not the result of individual choice. The mirror is only from the guide and the interaction of internal spirit of the world, and finally forms our cognitive field.
\end{abstract}

Keywords: Dynamic Psychology, Characterization, Ideology

\section{Introduction}

In real life, group perception mostly relies on the conceptual understanding of subjective logic, processing the external information through the sensory system to obtain the subject experience needed at present. Generally speaking, people are indifferent to the order of external things. Only when information ACTS directly on the host can individuals send signals to other living things to indicate their position. As the spiritual illusion of the "self" in the group, individual existence is only a continuation of the space-time memory, belonging to the reality of the moment, rather than the actual individual. At the same time, the subject experience of conditioned reflexes is also relatively existing, which changes at any time according to the increase of experience, while the audience's interpretation of things in the closed field is easily influenced by the opinions of communicators, and the public opinion environment in the later period will affect the seeds in the initial dissemination of mind.

\section{Gradual Change of Social Signs Shows the Spiritual Power of the "Trinity"}

Social identity originally refers to the special product of The Times, but it is extended here as the life concept in the collective memory of human beings. It records the growth process of a generation and is also the "source" that people absolutely believe in existence. The external expression of life exists in the way of material movement, while the "source power" of movement comes from the foundation of national belief, or the spiritual phenomenon of "trinity" of family concept, personal feeling and social ideal. Emile Durkheim argued that "collective expression is the result of extensive cooperation, which extends not only to space but also to time; 
Various minds unite, unite, and combine to form their ideas and feelings, to form these manifestations." [1]. Family concept is the most primitive symbol of contemporary people, even if the language is not, can also feel the affinity of cultural homology in similar areas. In the old east, family values were often an extension of religious experience in secular society. For example, Chinese Confucianism likes to use "benevolence, righteousness, propriety, wisdom and faith" to determine what a moral person should look like. Moreover, the family concept is also a continuation of life. As long as similar concepts or traditions are still effective in life, the ancestors must exist in the form of value intention.

Personal feelings are the comprehensive arrangement of individual feedback information in the society and the unity of causes and conditions in the complex social network. Needless to say, feelings are divided into internal experience and external feedback. Inner experience focuses on the empirical recall of one's own experience, and reestablishes one's own values or changes one's existing attitude based on an event or segment that has happened, so as to realize the revision of personality. Prince points out that "people with very different cultures often acknowledge that certain sets of identical elements are narratives and others are not, and they often tell whether or not the same narrative is true." [2]. It can be seen that different narrative methods and cultural habits form cultural barriers that are difficult for us to cross. Different from this, external feedback pays more attention to the immediate response to the external information, and indirectly penetrates the ideas and thoughts expressed by the external information into personal feelings, thus forming emotional or value identification and gradually forming a consensus from the outside to the inside. In today's multicultural society, the information brought by external feedback has controlled the thoughts and emotions of most people. How to awaken the inner experiential understanding of cultural connotation will become the key to the national issue.

Social ideal is the compound expectation of subjective initiative, which comes from the former two and greatly affects people's mental state. Generally speaking, personal ideal is the pursuit of unfulfilled wishes, while social ideal focuses on the desire of most people for "truth, goodness and beauty". Perhaps the individual ideal is often the sublimation of egoism, and those who emphasize the social ideal prefer to use a seemingly grand, but actually vague concept to express the ideal appeal of the public. In "the mob," le pen describes it as "he/it evokes visions of grandeur, and it is their vagueness that gives them their mystical power." Although this power seems to be enormous, "the group thinks with images, and images themselves will immediately lead to a series of images that have no logical relationship with it" [3]. Therefore, the social ideal is the blueprint of The Times. No one can say what it must be, but a steady stream of people wants to describe it.

To sum up, the continued concept of family, subjective personal feeling and grand social ideal "trinity" of life constitute the "initial impression" of human interpretation of things. If the deviation of the public's concept of family, personal feeling and social ideal occurs, then it will inevitably aggravate the situation of representation crisis. Social researchers need to explore a path from the tradition to the future, from the empirical self to the contemporary self.

\subsection{Meaning in Interactive Communication}

The positivist ethnography holds that "in the way of reflection meaning is seen as an object, a person, an idea, or an event placed in the real world, language ACTS as a mirror, reflecting the real meaning as if it were already there." [4]. Although language has the function of reflecting meaning, what is described by language is limited to the perspective that can be observed by the party concerned, or the meaning expressed by any language is incomplete and one-sided, and can only exist as a special meaning unit in space-time. For example, in the principles of semiotics, it is proposed that language is composed of two axes. The second one is the associative plane. Roland Barthes believed that "in the place of speech, units which have certain commonalities with each other are connected in human memory and form phrases () governed by various relations." [5].

Vocabulary is people's description of the phenomenon that has happened, meaning to let others know the state of the time as close as possible. This kind of "representation" has a certain guiding effect on subsequent cognition, but due to their different circumstances, readers will be restricted by conventional thinking in personal interpretation. In order to strengthen the social group's fundamental identification of the same thing, it is necessary to adopt relevant institutional control method, that is, "how to explain a certain behavior in a specific context" [6]. Traditional concepts unconsciously affect People's Daily life, while language is controlled by the conventional concepts. For example, Oriental people have the national complex of seeking good and avoiding evil, and call breaking things "peace of the year" to ease the unnecessary embarrassment under the current situation, while westerners like to regard broken things as a new beginning. Thus, it can be seen that even if the behavior of the same event is similar, due to different ethnic backgrounds, it will often be given a special meaning that it does not have. This meaning is made up of three parts: national belief, national ethics and national communication.

National belief is a kind of unrealized environmental choice that the group pursues together [7]. It is the spiritual power that people rely on. It also includes personal belief formed by imagination, observation and reflection. All of these can be attributed to the spiritual god for the image of one's desire to achieve but impossible to achieve. The significance of this "god" is to stimulate subjective initiative, and its unstable factors are caused by cultural conflicts. National ethics, which originally refers to those moral sentiments that need to be regulated, actually refers to the traditional concepts hidden in human consciousness in cultural transmission, and plays a role in explaining culture objectively. If the intention of the disseminator can be infiltrated into the shaping of ethics, all the explanations of later generations will be controlled by the ethical situation. Ethnic communication, also known as cultural 
integration, means regulating the values that people focus on in different cultural regions, the exchange of spiritual production, and the free selection and inclusiveness of cultural patterns.

To study the purpose of interactive communication, the key is to clarify the influence of various subconscious minds on current behaviors in a society with complicated causes and conditions, to explore the real intention of subconscious minds to express in communication, and to transform language conditions to control the whole process of cultural communication in the subsequent context. Both the media and the state and social groups need to deal with the popular consciousness in the minds of the public in a timely manner, and use similar information known by the public to bridge the gap that may exist between the national consciousness, the group consciousness and the individual consciousness.

\subsection{Implicative Non-logical Virtual Construction}

First, Roland Barthes believed that "ideology is the form of signification, while rhetoric is the form of signification". In order to further understand the attitude of mainstream ideology on social issues, it is necessary to further analyze the consciousness attribute of events themselves. Known as myths and legends, for example, it doesn't make much difference whether or not is almost as real life, but the myth of each nation's people believe that they are real, or is not set up events if lasted for a long time in the group, and the realistic pursuit of a problem with people have the same view, this event becomes cannot be falsified real events, as defined by Thomas theorem "if people define the condition is true, according to the result which is the true". Later generations can only develop according to similar patterns in ideology. If things go beyond the scope of specific description that people can imagine, even the truth cannot escape the fate of being questioned. From the perspective of history, there has never been any crisis in the representation of contemporary communication. However, when the new cultural concept transcends the traditional perspective of consciousness, the confrontation form of encoding and decoding will come again.

Second, "although it is impossible to be completely objective, we cannot use this as an excuse to let our emotions run free in unrestricted subjective situations [8]. Illogical fictions are rooted in daydream pleasures. Although people indulge in the dreams they weave and enjoy the things they can't get in reality but are fascinated by them, similar emotions are not universal and will eventually disappear in the waves of history. Most of the time, the irrational construction of individuals violates the real social order, and the public can be dismissive of it. However, the irrational construction of groups directly affects the social intention. After all, social development turns from irrational exploration to rational internal adjustment.

\section{Ideological Shift}

\subsection{Continuous Thinking and Reality}

The national consciousness penetrates into the collective thinking through study, life and work, and turns into the state of
"I" in the group assembly. In all the behavior process of "I" on the surface, the so-called "existence" is the continuation of the thoughts generated by the national complex. With "I" can feel everything is not actual, thinking of it is rooted in the national formulary spirit, although the social cultural codes can be used to it to spread some information, but in the "I" in the collective unconscious can't completely accept foreign elements, at best, is also the significance of the unreasonable to daily can refer to. Cultural crisis mostly in language and expression of fracture, is fundamentally different ideological mutual exclusivity, at any time as telling people "I" prototype, to step in processing, settlement readers personal emotional understanding, thus in the "reproduction" a particular need to restore the actual cognitive cultural meaning, namely the object of the original call.

\subsection{Implication of Irrational Rituals}

Irrational ritual is a very special technique in organizational communication, which can meet the needs of group members at various levels in a short time and release individual emotions. According to Arnheim, perception is a mode or pattern that has a relatively simple shape, consistent with the stimulus [9]. For example, in the mass held in church, the baptized will feel a special "divine grace" in it, temporarily confronting the complicated information of the external world, focusing on the sacred things, reveling in the melting consciousness and the gradual tranquility of emotions. Or in an assembly or ceremony, the host exerts a constant emotional orientation that leads the participants into an extreme state of being too happy or too sad, and then the people around them become infected with each other and start confiding in each other endlessly. In this process, the pre-consciousness will be affected by the fanatic emotion slowly subside, some of the unconscious memory with the needs of the host is awakened at any time; When the leader expresses his opinion, the participants are at the peak of emotional release and their thinking consciousness is extremely empty, so they lose their own ideas. They only recall similar events with the intention of the leader and strongly support the leader who awakens the collective consciousness through similar understanding.

Today's representational crisis reflects the growing individual consciousness of the people and the passing centralization consciousness of the authorities, and when the two fail to achieve dialogue, the official language of expression can no longer be a universal ideogram. Irrational rituals imply that they are intended to modulate the irrational ideas of the masses, and to awaken the deep memory of the group through some kind of movement [10]. For example, Schopenhauer believed that "rationality is feminine in nature, and it can only be given after it has received." In the control of ideology, irrationality is the soil for human development, while rationality is only the internal adjustment of society after the great change.

\subsection{Extension of "Natural" Memory}

In communication activities, although the public often corrodes the traditional representation with their own 
evaluation standards, "nature" still maintains the uniqueness of things through memory. Nature itself is a reality independent of consciousness, recording all actions within the range of human activities, the form closest to the original intention of life, and difficult to be obscured by the indicative tool of symbols; But "natural" memory transforms unspeakable emotions, allowing the meaning endowed by individual consciousness to spread to the public at the same time. For example, "dew from the white tonight, the moon is the hometown bright" in the "moon" refers to the beautiful things of hometown in the memory field, this kind of signifier and signifier in the official field has not been recognized by the system, but because people's memory structure in the association of the extension of the meaning to express. There is a supra-linguistic situation in the world. When a group conducts a series of activities around a symbol, the symbol not only represents the entity of the original reference, but also represents a memory representation.

Almost everything can be mechanically copied, only nature, the form closest to the original intention of life, is difficult to be covered by symbols as an indicative tool, or in other words, "nature" has always maintained its authenticity in the course of history, adding state attributes to all descriptive things. Benjamin sigh industrial bourgeois art of the era, is actually feeling is referred to in art form is being replaced by the mode of mechanical reproduction, the unique characterization are volume copy, he pointed out that "light failure from the two kinds of situations of the musical sound, they are related to the increase of public significance in contemporary life, namely modern mass to make objects in space and human nature are more likely to be close to the strong desire, just as they have to accept every physical copies to overcome the uniqueness of the strong tendency " [11]. Despite the public with their own evaluation standard eroding the characterization of traditional way, but the "natural" to a memory retains the unique things, no one can go beyond the "natural" and let things have a life, more not being "natural" alienation, after all, it's the language form, can change rather than memory hidden in the heart of the case.

"Nature" exists before consciousness, which can be converted into cognitive information. In the process of communication, "nature" is built on moving materials, and its vitality corresponds to the specific memory function of "nature". For example, in religious worship and team training, people cannot fail to use a large number of movements to strengthen their own ability, in the collective mutual super normal sports are often left a deep impression. Therefore, any "natural" use of memory requires intense physical activity, and when the behavior reaches unprecedented heights, out of excitement will not hesitate to remember what is going on. The most fanatical messages are not necessarily those with deep thoughts. What excites the whole nation is the group attention that makes people move from beginning to end.

\section{Represent Difficulties in Different Field}

\subsection{The Unconscious Presupposition of Individual Coding}

Representation is a process of "reconstructing" the world through discourse symbols and reinterpreting its meaning "16". In the process of global integration, different ideologies have raised dissenting voices in the interpretation of the world, and the symbolic meanings defined within nations and groups have been re-constructed. First of all, the spirit of The Times is divided into three parts by people of different ages. For example, the pre-modern thought that "seeing is believing, the view of truth has occupied most of the world in human history", while the modern thought that the existence of differences is legitimate, and different people have different opinions. Postmodernism believes that "there is no external world at all, everything exists within" [12]. Different thinking orientations lead to language differences. In addition, communication coding can also be affected by individual language, such as the popular Internet word "your sister", which is interpreted as "your sister" in root, but refers to the dirty word "your mother" in common language, which is often humorous in the younger generation. Obviously, the social unconscious infects people's language habits, and words that seem to be full of connotation may only be extensions of spoken words in personal digestion. The real meaning of a thing may lose its original function in the unconscious organization of an individual, and that new meaning comes from the habit of personal life.

\subsection{Complex Cognition of Phase Shift}

Vocabulary is not only a medium of expression, but also a tool of record in historical communication. However, historical records are often presented in different forms on the same thing. For example, the image of "dog" can be replaced by "dog" or "ground sheep", and the poet does not hesitate to choose the latter for the sake of rhyming. Although the traditional meaning of the original position of words has always been known to us, the loss of special expressions cannot make us feel the real history. Unitary vocabulary is broken in cognition, we must judge according to the purpose described in the realistic context, so as to link the reality of vocabulary with history.

In order to further explain the difference of cultural representation, we can explain the special meaning of things by means of language form. In the west "God" has a sacred meaning, the people put it in the heart to send a pious prayer; In the east, "god" is "man becomes god", and the public will use god to express the emotion of transcending themselves through efforts. The same things, however, have different referential effects because of cognitive differences, proving that concepts, "whatever their generality, are not the result of being placed in history or deposited in heavy collective habits by individuals" [13], or in addition to the speech of the speech situation affects the person's expression, and beyond personal consciousness of collective memories about the cultural 
representations.

\subsection{Dimension Cognition of Representational Legalization}

Representational legalization requires that words correspond to objectively existing things, while illegalization is the historical difference left by the audience's "atomism". As people for the interpretation of knowledge, "know is known, don't know is don't know." The former is to know the meaning of external knowledge, which belongs to the field of public knowledge; The latter is the inner spiritual level, belonging to the process of private understanding. According to the status of the two, knowledge and ignorance belong to the domain of knowledge, but the society can only recognize the effectiveness of the former in the public domain, but cannot make a unified judgment on its internal knowledge. Thus, it can be seen that representational illegalization is mainly caused by those inner knowledges that is not recognized to challenge public knowledge, or that public knowledge cannot meet the spiritual orientation of the public in a certain interval, so that the public chooses to use self-interpretation to overturn the traditional definition. Any information in the decoding, is difficult to get rid of the restriction of subjective experience, especially those "feeling", because the process contains many unstable factors, so finally aware of additional knowledge is the product of "good retribution", however, it is not a reality can support their opinions, but connected determines the imagination world.

In contemporary cultural representation, expression for the sake of living and expression for the sake of expression is an urgent concern for legalization. The expression of life is a different way according to its habitat. If the present state changes, the connotation of expression will be very different from the previous one, or this kind of cognition is the spirit endowed with. The expression for its own sake depends on the extension of the traditional idea, and what it wants to express is the directionality of the expression. Then Marcuse said that "in the human reality, all the beings who struggle to obtain the necessary conditions for their existence are therefore" unreal "and not free existence [14]. The crisis involves not only linguistic changes, but also psychological reconstruction and even ideological transformation. In order to further understand its generating factors and study the kinetic energy control of group psychology, it is a necessary way to effectively regulate group emotions and spread the main body value.

\section{Conclusion: The Exploration of the "Dynamic" Law of Group Psychology in Cultural Representation}

Cultural experience not only endows people with different temperament and temperament, but also creates a way for groups to explore the outside world. In other words, external ideas are not necessarily what human beings themselves believe to be the case. Although these ideas may have a short stay in the secular world, they are bound to become non-mainstream ideas due to historical choices. Therefore, any kind of similar cultural knowledge if not accepted by the public awareness and even, so this kind of similar things become deconstruction realistic concept of culture, although these fleeting concept in some extent by culture not entirely as a new idea, but unorthodox or countries do not admit things will eventually annihilate in a group of fanatical denigrated. Thus it can be seen that in the exploration of the imbalance of cultural representation, on the one hand, the difference of cultural experience can significantly reflect the status of individuals and create a unique perspective of observation in the process of interaction and communication; on the other hand, it may also cause unnecessary cultural conflicts, affect the establishment of mutual consensus and even form prejudice. Cultural conflict, of course, does not represent the existence of culture itself is meaningless, when people in history, or broader experience of yearning, the inherent cultural phenomenon is likely as the group's passion for virtual things by irreversible self-awareness into a herd of social self. In Lacan's opinion, the "social unconsciousness" was built on this track of denotative meaning. This article talked about family values, and social ideal is also relying on personal experience in the so-called noble desire of imagination.

According to the group psychological movement way, we can group psychological profiles of three states, one is for preliminary understanding the objective world, also is our psychological said on the sensory function of cultural landscape, the cultural landscape for the viewer, is the objective reflection of foreign culture in the main body experience, just like we traveling to different places, feel different from local humanistic amorous feelings; Secondly, for the depth of the subjective experience, or the process of self-understanding, this kind of cultural form is common in various religious activities, all of the people or things are calm as usual, but based on the experience of the believers subjective judgment, for an existing cultural significance of the secular, attach a's religious feelings cultural experience; A third is the "mirror image" to the society urgently mimicry, is the most superficial performance of followers for follow idol, a deeper step is about the capital of replication, namely social rhetoric of successful shape, as long as the individual's behavior is recognized by most people, then the mass to follow up on is the imprint of The Times. Therefore, "image generated by selecting the inner spiritual world experience of the world, rely on the public to participate in awakening experience interactive ceremony the main information in the world, while information is an extension of the" self "image in the communication, but the mirror is not the result of individual choice, is only from the guide and the interaction of internal spirit of the world, formed our cognitive field" [15]. Our pursuit of the balance of meaning in cultural representation is, in fact, to create a more orderly space for the spread of modern culture.

\section{Author Contributions}

Writing-original draft preparation and funding acquisition, 
Q. S.; writing-review and editing and supervision, H. W.

\section{Funding}

This research was funded by Study on the legalization and law of religious communication from the perspective of new media, grant number "18YBA430".

\section{Conflicts of Interest}

The authors declare no conflict of interest.

\section{References}

[1] Emile Durkheim. 2011. Les Formes Elementaires de la Vie Religieuse. Commercial Press. 18.

[2] Gerald Prince. 2013. Narratology The Form and Functioning of Narrative. Renmin university of China press. 79.

[3] Gustave Le Bon. 2005. The Crowd A Study of the popular mind. Central Compilation \& Translation Press. 25.

[4] Li Peng. 2013. Representation and decriminalization of Dual crisis. Modern communication 32-35. (In Chinese)

[5] Roland Barthes. 2009. Translated by Li Youzhen, Elements de semiology. Renmin university of China press. 54-57.
[6] Richard West/Lynn H. Turner. 2007. Introducing Communication Theory Analysis and Application. Renmin university of China press 122

[7] Max Weber. Max Weber Sociological anthology. people's publishing house. 2010. P166-173

[8] Geertz, C. Thick Description, inc. 1973. Geertz The Interpretation of cultures. New York, Basic Book 13-20.

[9] Rudolf Arnheim. 2001. Visual thinking, Sichuan people's publishing house. 36 .

[10] Schopenhauer ARTURE. 2007. Translated by Li Chengming, Schopenhauer's philosophy of life. Kyushu press 238.

[11] Walter Bendix Shoeflies Benjamin. Translated by Wan Caiyong by translation: 2001. The Art in the Age of Mechanical Reproduction. China city press 90.

[12] EARL Babble. translated by Qiu Zeqi. 2009. The Practice of Social Research. Huaxia press 10.

[13] Michel Foucault, Translated by Xie Qiang. 1998. L'Archeologie du Savoir. The joint publishing company LTD. 68.

[14] MARCUSE, H. Translation by Liu Ji. 2008. One Dimensional Man. Shanghai century publishing group 103.

[15] Song Quanhua, The spread of national mirror image from the perspective of Chinese culture. Southwest Jiaotong University. 2017. 1. (In Chinese). 